\title{
INSIST - IN Silico trials for treatment of acute Ischemic STroke
}

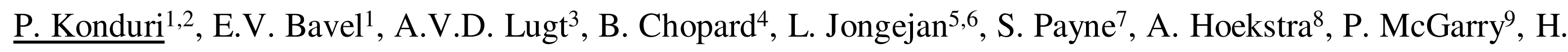
Marquering ${ }^{1,2}$, C. Majoie ${ }^{2}$

\section{ON BEHALF OF THE INSIST INVESTIGATORS}

${ }^{1}$ Academic Medical Center, Biomedical Engineering and Physics, Amsterdam, The Netherlands, ${ }^{2}$ Academic Medical Center, Department of Radiology and Nuclear Medicine, Amsterdam, The Netherlands. ${ }^{3}$ Erasmus Medical Center, Department of Radiology and Nuclear Medicine, Rotterdam, The Netherlands. ${ }^{4}$ Universite de Geneve, Computer Science Department, Geneve, Switzerland. ${ }^{5}$ Academic Medical Center, Center of Experimental and Molecular Medicine, Amsterdam. The Netherlands. ${ }^{6}$ Academic Medical Center, Experimental Immunology, Amsterdam, The Netherlands. ${ }^{7}$ The Chancellor-Masters and Scholars of the University of Oxford, Engineering Science- Institute of Biomedical Engineering, Oxford, United Kingdom, ${ }^{8}$ Universiteit van Amsterdam, Institute of Informatics- Faculty of Science, Amsterdam, The Netherlands. ${ }^{9}$ National University of Ireland, Biomedical Engineering, Galway, Ireland

\section{Introduction}

In-silico clinical trials are computer-based simulation of randomized controlled trials. INSIST is an international, inter-disciplinary consortium to develop models for in-silico Acute Ischemic Stroke (AIS) trials. This study aims to set up a platform to simulate randomized controlled trials to understand patient-specific (patho)physiology of AIS, interaction with treatment devices at relevant biological levels and treatment outcome.

\section{Design}

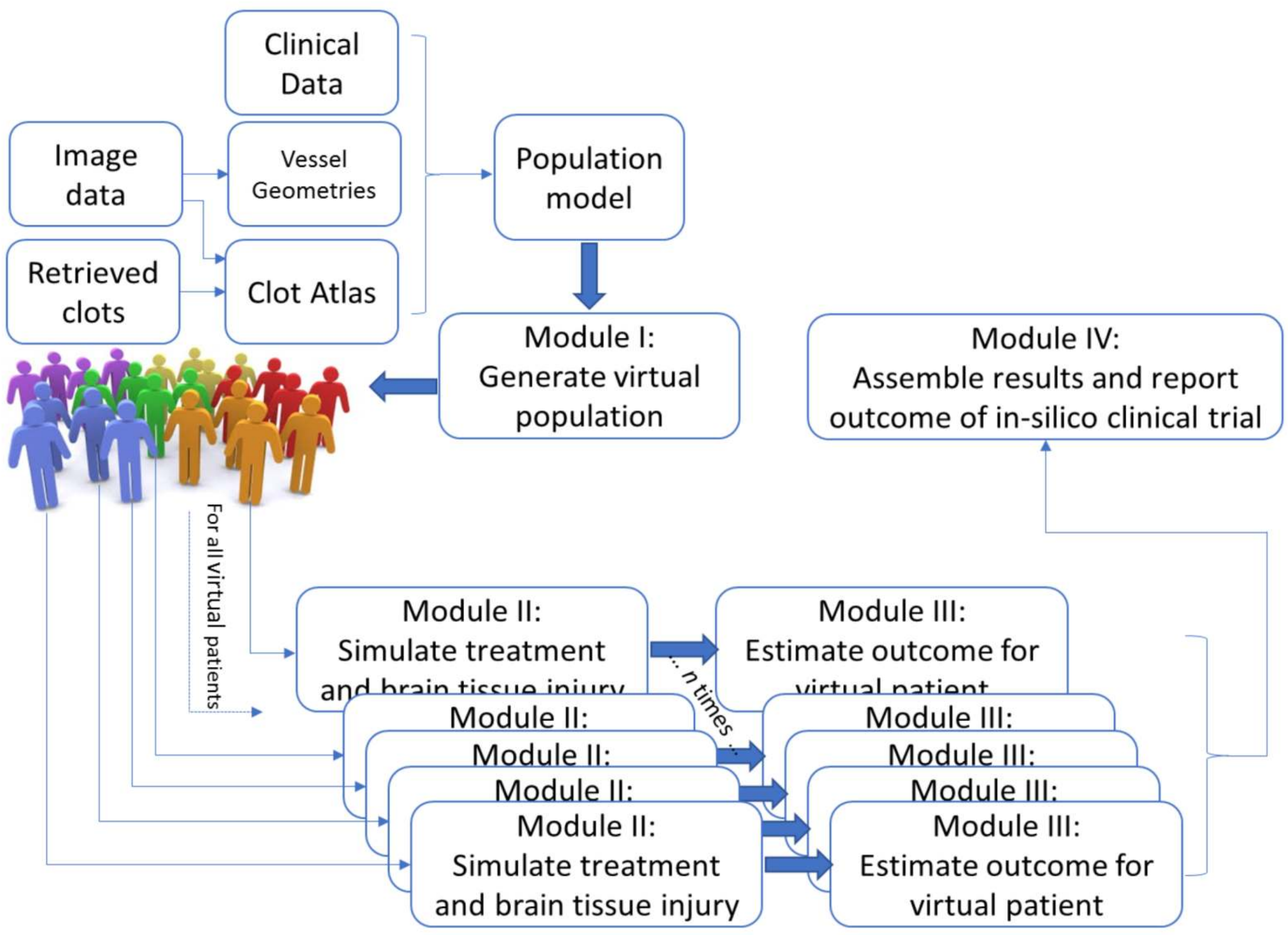

Figure: Schematic overview of a full in-silico clinical trial

\section{Outcome}

Accuracy of in-silico trials will be demonstrated by comparison with laboratory experiments and real-life randomised controlled trials.

\section{INSIST is funded under H2020. Grant:\#777072. www.insist-h2020.eu}

